\title{
CONCEPÇÃO DOS PROFISSIONAIS \\ DE SAÚDE ACERCA DO ABORTO PREVISTO EM LEI
}

Raissa Lorenna dos Santos Dantas¹, Maria Inês Bezerra de Melo², Laura Maria Tenório Ribeiro Pinto', Aniete Cintia de Medeiros Guimarães', Karla da Silva Ramos ${ }^{2}$

Objetivo: Descrever a concepção dos profissionais de saúde acerca do aborto previsto em lei. Metodologia: Estudo qualitativo de análise descritiva. Participaram 9 profissionais de nível superior preconizados pelas normas técnicas do Ministério da Saúde para o acolhimento de mulheres e gestantes vítimas de violência sexual em uma maternidade referência de Recife-PE. Resultados: Distanciamento de alguns profissionais no processo do abortamento por posicionamento ideológico e moral contrários, evidência da heterogeneidade dos profissionais acerca da temática. O desconhecimento dos profissionais sobre o funcionamento do serviço como referência da rede assistencial para acolhimento e atendimento às vítimas de violência sexual e interrupção de gravidez prevista em lei no município foram apresentadas como empecilho para o funcionamento do serviço. Conclusão: Percebe-se a necessidade de ampliar a compreensão acerca da assistência à mulher que vivencia o processo de abortamento, enfatizando os preceitos da atenção humanizada como preconizado pelo Ministério da Saúde, com o objetivo de melhorar a qualidade da assistência.

Descritores: Aborto legal, humanização da assistência, profissionais de saúde.

\section{DESIGN OF HEALTH PROFESSIONALS ABOUT LAW PROVIDED ABORTION}

Objective: To describe the conception of health professionals about abortion provided by law. Methodology: Qualitative study of descriptive analysis. Participants were 9 higher level professionals recommended by the technical norms of the Ministry of Health for the reception of women and pregnant women victims of sexual violence in a reference maternity hospital in Recife-PE. Results: Distancing of some professionals in the abortion process due to opposing ideological and moral positioning, evidence of the heterogeneity of the professionals about the theme. The professionals' lack of knowledge about the operation of the service as a reference of the assistance network for the reception and care of the victims of sexual violence and interruption of pregnancy provided for by law in the municipality were presented as an obstacle to the operation of the service. Conclusion: The need to broaden the understanding about the assistance to women experiencing the abortion process is emphasized, emphasizing the precepts of humanized care as advocated by the Ministry of Health, with the aim of improving the quality of care.

Descriptores: Legal abortion, humanization of care, health professionals.

\section{DISEÑO DE PROFESIONALES DE LA SALUD ACERCA DEL DERECHO ABORTO PROPORCIONADO}

Objetivo: Describir la concepción de los profesionales de la salud acerca del aborto previsto en la ley. Metodología: Estudio cualitativo de análisis descriptivo. Participaron 9 profesionales de nivel superior preconizados por las normas técnicas del Ministerio de Salud para la acogida de mujeres y gestantes víctimas de violencia sexual en una maternidad referencia de Recife-PE. Resultados: Distanciamiento de algunos profesionales en el proceso del aborto por posicionamiento ideológico y moral contrarios, evidencia de la heterogeneidad de los profesionales acerca de la temática. El desconocimiento de los profesionales sobre el funcionamiento del servicio como referencia de la red asistencial para acogida y atención a las víctimas de violencia sexual e interrupción de embarazo prevista en ley en el municipio fueron presentadas como impedimento para el funcionamiento del servicio. Conclusión: Se percibe la necesidad de ampliar la comprensión acerca de la asistencia a la mujer que vive el proceso de aborto, enfatizando los preceptos de la atención humanizada como preconizado por el Ministerio de Salud, con el objetivo de mejorar la calidad de la asistencia.

Descriptores: Aborto legal, Humanización de la assistência; Profesionales de salud. 


\section{INTRODUÇÃO}

No Brasil, o abortamento é crime tipificado pelo Código Penal, com exceção das situações previstas nos incisos I e II do artigo 128, que estabelece, desde 1940, o direito a este em casos de gravidez decorrente de estupro e quando há risco de morte para a gestante, sendo acrescido, pelo Superior Tribunal Federal (STF) no ano de 2012, em casos de bebês com anencefalia. A expressão "aborto previsto em lei" ou "aborto legal" significa a cessação da gravidez admitidos por lei $i^{(1,2,3,4)}$.

Apesar da legislação brasileira possibilitar o aborto previsto em lei desde 1940, o acesso aos serviços de saúde só foi regulamentado em 1989, quando surgiu o primeiro serviço de atendimento às mulheres vítimas de violência sexual no Hospital Municipal Artur Ribeiro de Saboya em São Paulo. Para ter acesso ao aborto, a mulher deveria apresentar a cópia do Boletim de Ocorrência (B.O.) e do laudo pericial do Instituto Médico Legal (IML). Através da Portaria MS/GM 1.508/2005, o B.O. deixou de ser exigido para o acesso aos serviços de aborto legal com a finalidade de minimizar o constrangimento e a dificuldade que muitas mulheres enfrentavam para realizar o procedimento(5).

Nesta perspectiva, o Ministério da Saúde lançou duas normas técnicas: Prevenção e Tratamento dos Agravos Resultantes da Violência Sexual contra Mulheres e Adolescentes e a Atenção Humanizada ao Abortamento, que orientam o atendimento dos profissionais de saúde no serviço público, tendo como objetivo padronizar a assistência e os procedimentos a serem adotados nesses $\operatorname{casos}^{(5,6,7)}$.

O aborto ser considerado crime, mas haver exceções à punição, gera uma ambiguidade penal, causando uma divergência no reconhecimento do direito ao aborto legal, não sendo a autonomia sexual e reprodutiva das mulheres o que se protege, mas a mulher vítima de estupro ou acaso da natureza, em relação à anencefalia fetal. A ilegalidade do aborto no país, além de todas as consequências danosas para a vida e a saúde das mulheres, também influi negativamente sobre os serviços de saúde, atingindo o atendimento realizado nos casos de aborto previsto em lei ${ }^{i(8,9)}$.

Um estudo com profissionais de saúde que atuam com o aborto legal demonstrou que ainda existe distância entre o que é previsto pelas políticas públicas e a realidade dos serviços de aborto legal no Brasil. Mesmo os serviços referências a esse atendimento, o cumprimento das recomendações contidas nas normas ainda é incerto(10).

Neste sentido, levando em consideração que a atitude dos profissionais de saúde pode ser um obstáculo importante para que as mulheres que cumprem os requisitos legais obtenham um aborto em hospital público, surgiu o seguinte questionamento: Qual a opinião de profissionais de saúde de uma maternidade de Recife diante das situações do aborto previsto em lei? Para tanto, buscou-se com este estudo descrever a concepção dos profissionais de saúde acerca do abortamento previsto em lei.

\section{METODOLOGIA}

\section{Tipo de estudo}

Trata-se de estudo qualitativo de natureza descritiva. Utilizou-se a abordagem da pesquisa qualitativa, com a finalidade de avaliar a compreensão subjetiva a respeito do assunto. Deste modo, enfatizando os significados e a compreensão que as pessoas atribuem às suas experiências do mundo social.

\section{Participantes da pesquisa}

A população do estudo foi composta por profissionais de saúde de nível superior. A amostra foi constituida por 9 (nove) profissionais de saúde de categorias diferentes, que incluem enfermeiros (as), médicos (as) obstetras, psicólogo e assistente social que atuam em uma maternidade do municipio de Recife, incluida na rede assistencial pela Secretaria Municipal de Saúde como referência para o acolhimento de mulheres e gestantes vítimas de violência sexual e interrupção da gravidez prevista em lei. Os critérios de inclusão dos participantes adotados foram: profissionais de saúde atuantes e responsáveis pelo acolhimento e atendimento de mulheres em situação de interrupção de gravidez prevista em lei, preconizados pelas normas técnicas do Ministério da Saúde.

\section{Local do estudo}

O estudo foi desenvolvido em uma maternidade de baixa complexidade onde oferece assistência integral à saúde da mulher, sendo referência para o atendimento às vítimas de violência sexual e interrupção de gravidez prevista em lei, no município de Recife/PE.

\section{Coleta dos dados}

A coleta de dados foi realizada durante o mês de janeiro de 2017, norteada por um questionário de autopreenchimento com questões semiestruturadas, enfocando as seguintes questões norteadoras: "Você é favorável a descriminalização do aborto?" "Você é favorável a realização do aborto previsto em lei?" "Na sua percepção quais os fatores atrapalham o andamento desse serviço?".

Para os profissionais que se propuseram a participar do estudo, foram solicitadas a leitura e assinatura do Termo de Consentimento Livre e Esclarecido - (TCLE), o qual foi assinado em duas vias, ficando uma via com a pesquisadora e a outra com a entrevistada. As entrevistas foram realizadas 
em locais próprios onde estivessem asseguradas condições mínimas de privacidade. Dada a homogeneidade da amostra, utilizou-se o critério de saturação de informação para a determinação do tamanho amostral.

\section{Análise dos dados}

Os dados coletados foram transcritos na integra. Após a análise textual discursiva, as falas dos participantes foram categorizadas por núcleos de sentido, na perspectivas da análise de conteúdo de Bardin, a qual configura-se como um conjunto de técnicas de análise das comunicações verbal ou não-verbal, que utiliza procedimentos sistemáticos e objetivos de descrição do conteúdo das mensagens ${ }^{(11)}$.

\section{Procedimentos éticos}

Em consonância à legislação que regulamenta a realização de pesquisas envolvendo seres humanos, incluídas na Resolução no 466 de 12 de dezembro de 2012 do Conselho Nacional de Saúde, o projeto de pesquisa foi submetido ao Comitê de Ética em Pesquisa em Seres Humanos do Instituto de Medicina Integral Professor Fernando Figueira (CEP-IMIP), aprovado com CAAE no 55474516.4.0000.5201 com número do protocolo: 1.603.027. Os envolvidos só foram incluidos na pesquisa após concordância voluntária e assinatura do Termo de Consentimento Livre e Esclarecido (TCLE).

O local da pesquisa foi referido pela letra $Q$ e os profissionais de saúde entrevistados encontram-se caracterizados conforme a ordem cronológica das entrevistas, sendo identificados pelas variações da letra $\mathrm{O}$ ( $\mathrm{Ol}$ a O9), com a finalidade de manter o anonimato da instituição e dos entrevistados.

\section{RESULTADOS}

A apresentação dos resultados desse estudo dividiu-se em quatro momentos: o primeiro refere-se à caracterização dos participantes da pesquisa, o segundo relacionado ao aborto previsto em lei: a dualidade de posicionamentos na prática profissional; o terceiro relacionado à descriminalização do aborto: por que se envolver?; e o quarto refere-se às nuances da prática assistencial frente ao aborto previsto em lei.

\section{Caracterização dos participantes da pesquisa}

O estudo foi constituido por 9 profissionais de saúde, que incluem enfermeiros(as), médicos(as) obstetras, psicólogo e assistente social que atuam em uma maternidade do município de Recife, incluida na rede assistencial pela Secretaria Municipal de Saúde como referência para o acolhimento de mulheres e gestantes vítimas de violência sexual e interrupção da gravidez prevista em lei.

No que se refere às idades dos participantes entrevistados, nota-se que a faixa etária varia entre 33 a 64 anos, sendo a média de 48 anos. Destes, 8 são do sexo feminino e 1 do sexo masculino. Quanto ao tempo de formação, este variou entre 7 e 30 anos, com média em 18 anos. Quanto à distribuição dos sujeitos por tempo de trabalho na unidade, nota-se que o tempo de inserção entre os entrevistados variou entre 6 meses e 26 anos.

\section{Aborto previsto em lei: a dualidade de posicionamentos na prática profissional}

O aborto previsto em lei é entendido por parte dos profissionais como um direito da mulher conquistado. No entanto, expõe-se distanciamento na participação de alguns profissionais nesse processo, e até mesmo "seletividade" de alguns casos, por posicionamento ideológico e moral contrário: "Cada caso deve ser visto e analisado individualmente. A pessoa envolvida tem a liberdade de decisão". (Q2); "Concordo nos casos de má formação com o objetivo que se dá e discordo em caso de estupro, já que existe a gravidez "saudável" e deveria seguir com apoio psicológico quando necessário". (Ol); "Acredito que a mulher em casos previsto em lei poderia decidir pela interrupção da gestação, mas como pessoa contra, não faço parte do processo de indução do aborto". (O5)

\section{Nuances da prática assistencial frente ao aborto previsto em lei}

A existência de algumas dificuldades vivenciadas diariamente pelos profissionais de saúde interfere no desenvolvimento da assistência ao aborto previsto lei, e por muitas vezes são consideradas como obstáculos para o funcionamento dos serviços.

A qualificação profissional neste âmbito apresenta-se deficiente. Os profissionais referem falta de atualizações do serviço em saúde, bem como, outros desconhecem sua própria função frente aos casos de assistência ao aborto previsto em lei, demonstrando uma necessidade de capacitação da equipe quanto à ampliação do conhecimento sobre a legislação, a garantia de direitos em saúde sexual e reprodutiva, e assistência em saúde: "Falta uma equipe especifica; falta maior divulgação na rede dos serviços de saúde obstétrica e mais investimentos estruturais e humanos". (Ol); "Falta de atualização profissional, protocolo préestabelecido na unidade, consenso entre a equipe de trabalho". (Q3); "Nunca participei dessa decisão, apenas realizo as ações de enfermagem". (08)

O desconhecimento dos profissionais acerca do próprio funcionamento do serviço, sendo considerado como referência da rede assistencial para o acolhimento e atendimento às vítimas de violência sexual e interrupção de gravidez prevista em lei no município de Recife/PE, se apre- 
senta como um empecilho na efetivação do processo de trabalho frente ao aborto previsto em lei: "Nunca presenciei, nem tive conhecimento de nenhum caso de abortamento legal neste serviço". (Q7); “...não percebo esse serviço com esse trabalho". (Q4); "Não reconheço essa maternidade como referência para esse tipo de atendimento". (Q6)

A infraestrutura dos serviços também emergiu como problema, expondo a ausência de espaços específicos para atendimento das mulheres que irão receber a assistência do aborto previsto em lei e sua internação conjunta após o procedimento com puérperas e recém-nascidos.:"Falta um setor específico para atender estas pacientes". (Ol)

\section{A descriminalização do aborto: por que se envolver?}

A descriminalização do aborto traz consigo uma vasta divergência de posicionamentos. A forma como o indivíduo se vê no mundo, suas concepções ideológicas, religiosas e politicas expõem-se como fatores que direcionam e definem decisões da vida cotidiana, bem como em relação ao objeto de estudo desta pesquisa.

Nesse âmbito, verificou-se a existência de profissionais de saúde que se mostram contra a prática do aborto, bem como, outros que defendem a descriminalização por entendê-la como um problema de saúde pública. Dessa forma, fica evidente a heterogeneidade de posições dos profissionais de saúde acerca desta temática: "Sou favorável, desde que seja em relação ao aborto responsável, nos casos previsto em lei, devidamente comprovados". (O3); "Creio que é uma escolha pessoal baseada nas experiências pessoais, visão de mundo e espiritualidade da mulher e não pode ser criminalizada e definida por outras pessoas". (Q7); “Sou contra ao aborto legal e a descriminalização do aborto, devido aos meus princípios religiosos". (Q9); Sou contra o aborto, mas acredito que a descriminalização irá reduzir a mortalidade materna". (O5)

\section{DISCUSSÃO}

O abortamento é um grave problema de saúde pública, está presente no cenário brasileiro, e é tema gerador de várias discussões, tanto pela defesa de sua legalização quanto pela manutenção, parcial ou irrestrita, de sua proibição. As influências de questões éticas, morais, socioeconômicas, políticas, culturais e religiosas trazem dificuldades ao seu enfrentamento. Nesse contexto, tornam-se necessárias reflexões acerca da autonomia da mulher sobre seu corpo, da visão do profissional de saúde diante de tais decisões, além das implicações ético-políticas para o campo da saúde pública(12,13).

O aborto previsto em lei, apesar de ser legalmente aceito, tem sido um tema que vem causando dualidade de posicionamentos na prática assistencial dos profissionais que lidam com essa questão. Os resultados deste estudo mos- tram que ainda existem alguns entraves para a adequada assistência às mulheres que vivenciam esse processo.

A discussão perpassa pela relação entre o direito e a moral. Por um lado, parte dos profissionais entendem o aborto previsto em lei como um direito garantido para todas as mulheres, enquanto que por outro lado, as crenças individuais e aspectos sócios culturais esbarram na efetivação desse direito garantido. Nesse sentido, surgem alguns obstáculos para a efetivação do funcionamento dos serviços do abortamento previsto em lei.

Corroborando com Madeiro (2016), o presente estudo evidenciou que dentre os entraves assistenciais, sobressai a necessidade de maior capacitação da equipe quanto à ampliação do conhecimento sobre a legislação e sobre a garantia de direitos em saúde sexual e reprodutiva, entendimento acerca de "violência de gênero", "humanização" e "direitos humanos"(10).

Em um estudo realizado por Rocha et al (2015), demonstrouse que os profissionais de saúde frequentemente são ambivalentes a respeito do tema "aborto", pois têm que enfrentar valores profissionais e morais conflitantes. Os casos de malformações incompatíveis com a vida são mais aceitos. No entanto, em situações de gravidez decorrente de violência sexual, em grande parte, mostram-se desfavoráveis, condizentes com alguns discursos do presente estudo. Esse aspecto confirma o peso da desconfiança e da suspeição como marcador da moralidade do profissional diante da mulher que procura atendimento para abortamento nos casos previstos em lei(9,13).

É oportuno expor que a necessidade de qualificação profissional é mencionada como um fator determinante para a efetivação da assistência à mulher que vivencia o processo de abortamento previsto em lei. Sabe-se que o profissional deve ter adequado conhecimento da legislação, treinamento em como atender a mulher sem julgamentos ou preconceitos, bem como ofertar apoio psicológico.

Cabe ressaltar que um dos escopos do Ministério da Saúde é justamente buscar promover a atenção qualificada e humanizada às mulheres em situação de abortamento. Para o profissional, a atenção humanizada implica uma conduta na qual deve abstrair suas convicções morais, culturais, religiosas, bem como outros aspectos que possam influir no atendimento à paciente; ou seja, sua atitude deve ser norteada, acima de tudo, pela imparcialidade (justiça). (7.13)

Outro fator a ser considerado como um entrave assistencial é o desconhecimento dos profissionais acerca do próprio funcionamento do serviço como referência da rede assistencial para o acolhimento e atendimento às vítimas de violência sexual e interrupção de gravidez prevista em lei no município de Recife/PE. A invisibilidade desses serviços dentro dos próprios hospitais e maternidades fora relatado 
em um dossiê acerca do aborto legal em hospitais públicos. O fato dos profissionais desconhecerem a existência do funcionamento desses serviços limita o acesso que as mulheres têm por lei desse direito garantido(10, 14, 15).

É notório que os serviços de referência para o atendimento integral às mulheres em processo de abortamento previsto em lei deveriam oferecer infraestrutura adequada. No entanto, no presente estudo, expôs-se a ausência de espaços específicos para atendimento das mulheres que irão receber a assistência do aborto previsto em lei bem como sua internação conjunta após o procedimento com puérperas e recém-nascidos. Esta circunstância também foi verificada por um estudo nacional acerca dos Serviços de aborto legal no Brasil(14, 16).

Assim, pensar em serviços com recursos de infraestrutura insuficientes é preceder a interferência no desenvolvimento das atividades cotidianas, pois o bom acolhimento e a satisfação com a assistência apresenta estreita relação com os espaços de produção dos cuidados e, consequentemente, se articula com a humanização das práticas em saúde.

Ainda, a presente pesquisa contemplou aspectos sobre a descriminalização do aborto, trazendo consigo divergências de posicionamentos. Alguns profissionais entrevistados colocaram-se a favor da descriminalização, fazendo referência aos direitos reprodutivos e sexuais das mulheres, à autonomia destas sobre seu próprio corpo, à necessidade da redução de mortalidade materna nesse âmbito. No entanto, outros demonstraram-se contra, explicitando a interferência de fatores ético-profissionais e/ ou pessoais-religiosos durante o processo decisório da interrupção da gestação.

Posicionar-se diante do aborto como um direito da mulher ao controle de seu próprio corpo deveria ser uma questão de saúde pública. $O$ processo decisório sobre escolher interromper ou não a gestação deveria ser da mulher, sob a perspectiva dos direitos sexuais e reprodutivos bem como da autonomia sobre seu próprio corpo. Por outro lado, posicionamentos contrários à legalização, comumente, direcionam o entendimento e as medidas a serem tomadas, para o campo da moralidade e do individualismo, deixando de considerar o contexto sociocultural e as relações de gênero e sociais que estão imbricadas na prática do aborto ${ }^{(16,17)}$.

\section{Limitações do estudo}

De modo geral, a escassez de publicações atualizadas acerca dos serviços de abortamento legal limitou a discus- são do estudo. Isso sugere que por ser uma temática que envolve questões paradigmáticas da bioética o tema ainda é visto com cautela por estar vinculado a uma das legislações mais restritivas nos casos de aborto no mundo.

\section{Contribuições do estudo para a prática}

No âmbito científico, este estudo permite ampliar da compreensão acerca da assistência à mulher que vivencia o processo de abortamento, enfatizando os preceitos da atenção humanizada como preconizado pelo Ministério da Saúde, propiciando a melhoria da qualidade assistencial.

\section{CONCLUSÃO}

O desenvolvimento da assistência à mulher que vivencia o abortamento previsto em lei perpassa por diversas influências de matizes ideológicas. Dessa forma, os discursos dos profissionais obtiveram variados sentidos no que se refere aos aspectos que englobam o processo assistencial nesse âmbito.

Nesse estudo, ficou evidente que o aborto previsto em lei é entendido por parte dos profissionais como um direito da mulher conquistado. No entanto, expõe-se distanciamento na participação de alguns profissionais nesse processo por posicionamento ideológico e moral contrários.

A existência de algumas dificuldades vivenciadas diariamente pelos profissionais de saúde, interferem no desenvolvimento da assistência ao aborto previsto em lei, e por muitas vezes são consideradas como obstáculos para o funcionamento dos serviços. A qualificação profissional nesse âmbito apresenta-se com uma lacuna, visto que os profissionais referem falta de atualizações do serviço em saúde, bem como, outros desconhecem sua própria função frente aos casos de assistência ao aborto previsto em lei.

Diante dos resultados da pesquisa, fica evidente a heterogeneidade de posições dos profissionais de saúde acerca da descriminalização do aborto. Verificou-se ainda a existência de profissionais de saúde que se mostram contra a prática do aborto, porém defendem sua descriminalização por entendê-lo como um problema de saúde pública.

\section{Contribuição dos autores:}

Concepção e/ou desenho, análise e interpretação dos dados, redação do artigo, revisão crítica, revisão final: Raissa Lorenna dos Santos Dantas, Aniete Cintia de Medeiros Guimarães, Karla da Silva Ramos, Laura Maria Tenório Ribeiro Pinto, Maria Inês Bezerra de Melo. 


\section{REFERÊNCIAS}

1. Benute GRG, Nonnenmacher D, Nomura RMY, Lucia MCS, Zugaib M. Influência da percepção dos profissionais quanto ao aborto provocado na atenção à saúde da mulher. Rev. Bras. Ginecol. Obstet. [Internet]. 2012 [cited 2019 Jul 15]; 34(2): 69-73. Available from: http://www. scielo.br/pdf/rbgo/v34n2/a05v34n2.pdf

2. Brasil. Presidência da República. Casa Civil. Decreto Lei n. 2.848 , de 7 de dezembro de 1940. Código Penal. Diário Oficial da União 1940; 31 dez.

3. Supremo Tribunal Federal. Arguição de Descumprimento de Preceito Fundamental n. 54 [internet]. 2012 [cited 2018 jul 1]. Available from: http://www.stf.jus.br/portal/diarioJustica/verDiarioProcesso.a sp?numDj=77\&dataPublicacaoDj=20/04/20128incidente $=22269548 \mathrm{c}$ odCapitulo=2EnumMateria=10\&codMateria $=4$.

4. Ribeiro CL, de Oliveira Albuquerque F, \& de Souza AR. Internações por aborto espontâneo: um retrato de sua ocorrência em Fortaleza. Enferm Foco [Internet]. 2017 [cited 2019 Jul 10]; 8(1),37-41. Available from: http:// revista.cofen.gov.br/index.php/enfermagem/article/view/584/365

5. Colás OR, Andalaft-Neto J, Rosas CF, Kater JR, Pereira IG. Aborto legal por estupro - primeiro programa público no país. Bioética 2009: 2(1):81-85.

6. Possolo CP, de Souza Silva GT, Rechmann IL, Nazar Machado MB Chaves SM, Magalhães TA., \& Vásquez VDL. Disciplina normativa do atendimento às vítimas de violência sexual à luz da lei $n .912 .845 / 2013$ Rev Direito UNIFACS - Debate Virtual [Internet]. 2014 [cited 2017 jun 1]; 163. Available from: https://revistas.unifacs.br/index.php/redu/article/view/2921

7. Brasil. Ministério da Saúde. Secretaria de Atenção à Saúde. Atenção humanizada ao abortamento: norma técnica. $2 a$ ed. Brasilia: Ministério da Saúde [Internet]. 2005. [cited 2019 Jun 12]; 4: 32. Available from:http://bvsms.saude.gov.br/bvs/publicacoes/atencao_humanizada_abortamento.pdf

8. Brasil. Ministério da Saúde. Secretaria de Atenção à Saúde. Prevenção e tratamento dos agravos resultantes da violência sexual contra mulheres e adolescentes: Norma Técnica. Brasilia: Ministério da Saúde; [Internet]. 2012 [cited 2019 Jul 1]; 3a ed. atual. ampl. Available from:http://bvsms.saude.gov.br/bvs/publicacoes/prevencao_agravo_violencia_sexual_mulheres_3ed.pdf
9. Diniz D, Dios VC, Mastrella M, Madeiro AP. A verdade do estupro nos serviços de aborto legal no Brasil. Rev. Bioét. [Internet]. 2014 [cited 2019 Jul 10]; 22(2): 291-298. Available from: http://www.scielo.br/pdf/ bioet/v22n2/11.pdf

10. Madeiro AP, Diniz D. Serviços de aborto legal no Brasil - um estudo nacional. Ciênc. saúde coletiva [Internet]. 2016 [cited 2019 Jul 23]; 21( 2 ): 563-572. Available from: http://www.scielo.br/scielo. php?script=sci_arttextEpid=S1413-81232016000200563\&lng=en.

11. Bardin L. Análise de conteúdo. São Paulo: Edições 70, 2011, 229 p.

12. Santos VC, Anjos KF, Souzas R, Eugênio BG. Criminalização do aborto no Brasil e implicações à saúde pública. Rev. Bioét. [Internet]. 2013 [cited 2019 Jul 23]; 21(3): 494-508. Available from: http://www. scielo.br/pdf/bioet/v2ln3/al4v2ln3.pdf

13. Rocha WB; Silva AC, Leite SM L., Cunha R. Percepção de profissionais da saúde sobre abortamento legal Rev. Bioét. [Internet]. 2015 [cited 2019 Jun 10]; 23(2): 387-399. Available from: http://www.scielo. br/pdf/bioet/v23n2/1983-8034-bioet-23-2-0387.pdf

14. Talib RA, Citeli MT. Serviços de aborto legal em hospitais públicos brasileiros (1989-2004) - Dossiê. Católicas pelo Direito de Decidir [Internet]. 2005 [cited 2019 Jun 13]. Available from: http://www. bibliotecadigital.abong.org.br/bitstream/handle/11465/305/CDDBR_servi\%c3\%a7o_aborto_legal_hospitais_publicos_brasileiros. pdf?sequence $=1$ EisAllowed $=y$

15. Diniz D, Medeiros M, Madeiro A. Pesquisa Nacional de Aborto 2016. Ciênc. saúde coletiva [Internet]. 2017 [cited 2019 Jun 10]; 22(2): 653-660. Available from: http://www.scielo.br/pdf/csc/v22n2/14138123-csc-22-02-0653.pdf

16. Farias RS, Cavalcanti LF. Atuação diante das situações de aborto legal na perspectiva dos profissionais de saúde do Hospital Municipal Fernando Magalhães. Ciênc. saúde coletiva [Internet]. 2012 [cited 2019 Jun 10], 17(7): 1755-1763. Available from: http://www.scielo.br/ pdf/csc/v17n7/14.pdf

17. Prata MCS, Campos RSP, Souza LBLN, Fernandes GL, Hime LFCF Abortamento entre mulheres vitimas de violência sexual: conflitos e desafios. Rev Aten Saúde [Internet]. 2015 [cited 2019 Jun 10]; 13(46): 106-112.Available from: file:///C:/Users/WINDOWS/Downloads/346412828-1-PB.pdf

RECEBIDO EM:24/01/2018.

ACEITO EM:16/04/2019

160 | Enferm Foco [Internet]. 2019;10(2): 155-160. 